J. Nonlinear Var. Anal. 5 (2021), No. 6, pp. 893-907

Available online at http://jnva.biemdas.com

https://doi.org/10.23952/jnva.5.2021.6.04

\title{
ON VARIATIONAL-HEMIVARIATIONAL INEQUALITIES WITH NONCONVEX CONSTRAINTS
}

\author{
OUAYL CHADLI ${ }^{1}$, JEN-CHIH YAO $^{2, *}$ \\ ${ }^{1}$ Department of Economics, Ibn Zohr University, B.P. 8658, Poste Dakhla, Agadir, Morocco \\ ${ }^{2}$ Department of Mathematics, Zhejiang Normal University, Jinhua 321004, China
}

\begin{abstract}
In this paper, we consider a nonconvex constrained variational-hemivariational inequality problem for a star-shaped set. The existence of solutions is shown by an approach based on the equilibrium problem theory, and a penalization method. An application to a nonconvex constrained semipermeability model for the stationary heat conduction problem is provided. Our results are new and improve considerably recent results in literature.
\end{abstract}

Keywords. Equilibrium Problems; Clarke subgradient; Hemivariational inequality; Maximal monotone bifunction; Pseudomonotone bifunction.

\section{INTRODUCTION}

Variational and hemivariational inequalities have been extensively investigated in the last several decades and are widely used in the study of many nonlinear boundary value problems with a large number of applications in contact mechanics and engineering; see, e.g., [1, 2, 3, 4] and the references therein. The theory of variational inequalities was developed in early sixty's by using the arguments of monotonicity and convexity, including the properties of the subdifferential of a convex function. Hemivariational inequalities are generalizations of variational inequalities and their origin is in nonsmooth mechanics. They were introduced and studied first by Panagiotopoulos in order to model problems in mechanics involving multivalued and nonmonotone constitutive laws and boundary conditions. These multivalued relations are derived from nonsmooth and nonconvex superpotentials by using the generalized gradient of Clarke.

Variational-hemivariational inequalities represent a special class of inequalities, in which both convex and nonconvex functions are present. We refer to the book [5] where the existence, uniqueness, and convergence results for various class of variational-hemivariational inequalities were studied, as well as the applications of these inequalities in the study of mathematical models which describe the contact between a deformable body and a foundation. Recently, semicoercive variational-hemivariational inequalities were studied via regularization techniques, and used to model unilateral contact problems with nonmonotone boundary conditions as well as the delamination of composite structures with a contaminated interface layer; see, e.g., [6, 7] and the references therein.

${ }^{*}$ Corresponding author.

E-mail addresses: o.chadli@uiz.ac.ma (O.Chadli), jen-chih.yao@zjnu.edu.cn (J.-C. Yao).

Received March 30, 2021; Accepted July 31, 2021.

(c)2021 Journal of Nonlinear and Variational Analysis 
In this paper, we study the existence of solutions of a class of variational-hemivariational inequalities in which the set of admissible elements is not necessarily convex. We consider the case where the constrained set is star-shaped with respect to a ball. Hemivariational inequalities with nonconvex constrained set were studied earlier for stationary problems by Naniewicz [8], Naniewicz and Panagiotopoulos [2, Section 7.4], Goeleven [9], and Goeleven [10] in a noncoercive framework. Evolutionary constrained problems on star-shaped sets were studied in $[11,12]$. Most of the techniques used in the contributions above $([2,8,9,11,12])$ are based on a surjectivity theorem for multivalued pseudomonotone operators in reflexive Banach spaces, and a penalization method, in which the small parameter does not have to tend to zero. Very recently, Migórski and Fengzhen [13] studied variational-hemivariational inequalities on nonconvex starshaped sets by using the method based on the KKM (Knaster-Kuratowski-Mazurkiewicz) theory.

In this paper, we use a new method based on the theory of equilibrium problems. Mathematically, an equilibrium problem (for short, (EP)) [14] can be considered as a generalization of many mathematical models, such as variational inequality problems, optimization problems, fixed point problems, complementarity problems, minimax inequalities, and Nash equilibrium problems; see, e.g., $[15,16,17,18,19]$ and the references therein. It is well known that a hemivariational inequality is no longer a variational inequality due to the absence of the convexity of the superpotential functional in its expression. The equilibrium problem formulation seems to be a very powerful tool to study hemivariational inequalities in the sense that the techniques for solving variational inequalities could be used to study hemivariational inequalities through an equilibrium formulation. In fact, equilibrium problems lead to a common formulation of variational and hemivariational inequalities.

In our approach, we use a penalization method in which a small parameter does not have to tend to zero. The penalized problem is studied by using the recent results in [20] on mixed equilibrium problems. They were described by the sum of a maximal monotone bifunction in the sense of Blum-Oettli [14] and pseudomonotone bifunction in the topological sense, that is, in the sense of Brézis. Regarding the maximal monotonicity, we refer to Blum-Oettli [14] wherein this condition on bifunctions was firstly introduced as an extension of the corresponding one of operators; see Hadjisavvas-Khatibzadeh [21] for more developments on this property. The notion of the pseudomonotonicity for bifunctions in topological sense was considered by Gwinner [22] and it is motivated by the concept of a topological pseudomonotone operator in the sense of Brézis [23]. Our results are new and considerably improve the results obtained in [13]. We provide an application to a nonconvex constrained semipermeability model for the stationary heat conduction problem. By our approach, we considerably relax the assumptions on a similar problem treated in [13].

\section{PRELIMINARIES}

Given a reflexive Banach space $X$ with the norm $\|\cdot\|_{X}$, its topological dual space is denoted by $X^{*}$, and the duality pairing of $X$ and $X^{*}$ is denoted by $\langle\cdot, \cdot\rangle_{X}$. For each subset $M$ of $X$, we denote by $\operatorname{conv}(M)$ the convex hull of $M$, and by $\bar{M}$ the closure of $M$ in $X$. We shall use $\mathscr{F}(M)$ to denote the family of all finite subsets of $M$. We write " - " to denote the weak convergence and " $\rightarrow$ " to denote the strong convergence in $X$. By $\bar{B}\left(u_{0}, \rho\right)$, we denote the closed ball in $X$ 
with center $u_{0} \in X$ and radius $\rho>0$, i.e.,

$$
\bar{B}\left(u_{0}, \rho\right)=\left\{u \in X:\left\|u-u_{0}\right\|_{X} \leq \rho\right\} .
$$

Definition 2.1. Let $C$ be a subset of $X$. We say that $C$ is star-shaped with respect to a ball $\bar{B}\left(u_{0}, \rho\right)$ if

$$
t u+(1-t) v \in C \text { for all } u \in C, v \in \bar{B}\left(u_{0}, \rho\right), t \in[0,1] .
$$

In the sequel, we simply say that a set $C$ is star-shaped if it is star-shaped with respect to a closed ball.

For the convenience of the reader, we recall the Clarke's generalized derivative and the generalized gradient for a locally Lipschitz function.

Definition 2.2. [24] Let $X$ be a Banach space, and $g: X \rightarrow \mathbb{R}$ be a locally Lipschitz function, that is, for every $x \in X$, there exist a neighbourhood $\mathscr{N}$ of $x$ and a constant $k_{x}>0$ such that $|g(z)-g(y)| \leq k_{x}\|z-y\|_{X}$ for all $z, y \in \mathscr{N}$. The Clarke's generalized directional derivative of $g$ at the point $x \in X$ in the direction $w \in X$ is defined by

$$
g^{0}(x ; w):=\limsup _{y \rightarrow x, t \downarrow 0} \frac{g(y+t w)-g(y)}{t} .
$$

The generalized gradient of $f$ at $x \in X$ is defined by

$$
\partial g(x):=\left\{x^{*} \in X^{*}: g^{0}(x ; w) \geq\left\langle x^{*}, w\right\rangle, \forall w \in X\right\} .
$$

The next two Lemmas give the important properties of the Clarke's generalized directional derivative and the generalized gradient.

Proposition 2.1. [24, Proposition 2.1.1] Let $g: X \rightarrow \mathbb{R}$ be a locally Lipschitz functional of constant $k_{x}$ near the point $x \in X$. Then,

(i) the function $w \in E \mapsto g^{0}(x ; w)$ is finite, positively homogeneous, sub-additive and satisfies

$$
\left|g^{0}(x ; w)\right| \leq k_{x}\|w\|_{X}
$$

(ii) $g^{0}(x ; w)$ is upper semicontinuous as a function of $(x, w)$.

Proposition 2.2. [24, Proposition 2.1.2] Let $g: X \rightarrow \mathbb{R}$ be a locally Lipschitz functional of constant $k_{x}$ near the point $x \in X$. Then,

(i) $\partial g(x)$ is a convex, weak ${ }^{*}$ compact subset of $X^{*}$ and

$$
\left\|x^{*}\right\|_{X^{*}} \leq k_{x}, \text { for all } x^{*} \in \partial g(x) ;
$$

(ii) for each $w \in X$, one has

$$
g^{0}(x ; w)=\max \left\{\left\langle x^{*}, w\right\rangle: x^{*} \in \partial g(x)\right\} .
$$

For a nonempty $C \subset X$, by $d_{C}: X \rightarrow \mathbb{R}$, we denote the distance function of $C$ defined by

$$
d_{C}(u)=\inf _{v \in C}\|v-u\|_{X}, \text { for all } u \in X .
$$

The Clarke's generalized directional derivative $d_{C}^{0}(u ; v)$ is well defined for $u, v \in X$ since $d_{C}$ is Lipschitz continuous.

In the sequel, we shall need the following discontinuity property of the Clarke directional derivative of the distance function for a star-shaped set; see [25, Lemma 7.2]. 
Lemma 2.1. Let $X$ be a reflexive Banach space, and let $C \subset X$ be a closed set which is starshaped with respect to a ball $\bar{B}\left(u_{0}, \rho\right)$ for some $u_{0} \in C$ and $\rho>0$. Then,

$$
\begin{aligned}
& d_{C}^{0}\left(u ; u_{0}-u\right) \leq-d_{C}(u)-\rho, \text { for all } u \notin C, \\
& d_{C}^{0}\left(u ; u_{0}-u\right)=0, \text { for all } u \in C .
\end{aligned}
$$

Next we recall the definitions and properties of cones in a Banach space $X$. Let $C$ be a subset of $X$ and $u \in C$. The Bouligand (contingent) cone to $C$ at $u$ is defined by

$$
K_{C}(u)=\left\{v \in X: \liminf _{t \downarrow 0} \frac{d_{C}(u+t v)}{t}=0\right\} .
$$

The set $K_{C}(u)$ is a closed cone and it can be equivalently defined as follows

$$
K_{C}(u)=\left\{v \in X: \exists\left\{v_{k}\right\} \subset X \text { and }\left\{t_{k}\right\} \subset(0,+\infty) \text { s.t. } v_{k} \rightarrow v, t_{k} \rightarrow 0 \text { and } u+t_{k} v_{k} \in C, \forall k \in \mathbb{N}\right\} .
$$

The Clarke's tangent cone to $C$ at $u$ is defined by

$$
T_{C}(u):=\left\{v \in X: d_{C}^{0}(u ; v)=0\right\}=\left\{v \in X: d_{C}^{0}(u ; v) \leq 0\right\} .
$$

The set $T_{C}(u)$ is a closed cone and we have the following equivalent definition:

$$
T_{C}(u)=\left\{v \in X: \forall u_{k} \rightarrow u, u_{k} \in C, \forall t_{k} \searrow 0, \exists v_{k} \rightarrow v \text { such that } u+t_{k} v_{k} \in C, \forall k \in \mathbb{N}\right\} .
$$

Note that $T_{C}(u) \subset K_{C}(u)$. If $T_{C}(u)=K_{C}(u)$, then we say that $C$ is regular at the point $u \in C$. If $C$ is closed, convex, and $u \in C$, then $C$ is regular at $u$; see [25, Theorem 10.39]. Moreover, if $C$ is a convex set in $X$ and $u \in C$, then $K_{C}(u)$ is convex and $C \subset u+K_{C}(u)$; see [25, Proposition 2.9].

We recall also the following concepts, which are useful in the next section.

Definition 2.3. A single-valued mapping $A: X \rightarrow X^{*}$ is said to be

(i) monotone if $\langle A(u)-A(v), u-v\rangle_{X} \geq 0$ for all $u, v \in X$;

(ii) maximal monotone if $A$ is monotone and $\left\langle v^{*}-A(u), v-u\right\rangle_{X} \geq 0$ for all $u \in X$ implies $A(v)=v^{*}$, i.e. $T$ has no proper extension;

(iii) pseudomonotone in the sense of Brézis (for short, B-pseudomonotone) if, for any sequence $\left\{u_{n}\right\}_{n \in \mathbb{N}} \subset X$ satisfying $u_{n} \rightarrow u$ in $X$ and $\lim \sup \left\langle A\left(u_{n}\right), u_{n}-u\right\rangle \leq 0$, we have

$$
\liminf \left\langle A\left(u_{n}\right), u_{n}-v\right\rangle_{X} \geq\langle A(u), u-v\rangle_{X}, \quad \text { for all } v \in X ;
$$

(iv) quasimonotone in the topological sense (for short, T-quasimonotone) if, for any sequence $\left\{u_{n}\right\}_{n \in \mathbb{N}} \subset X$ such that $u_{n} \rightarrow u$ in $X$, we have

$$
\limsup _{n \rightarrow \infty}\left\langle A\left(u_{n}\right), u_{n}-u\right\rangle_{X} \geq 0
$$

(v) demicontinuous if $u_{n} \rightarrow u$ in $X$ implies $A\left(u_{n}\right) \rightarrow A(u)$ in $X^{*}$;

(vi) hemicontinuous (respectively, upper hemicontinuous) if, for all $u, v, w \in X$, the functional $t \mapsto\langle A(u+t v), w\rangle_{X}$ is continuous (respectively, upper semicontinuous) on [0,1];

(vii) bounded if and only if $A$ maps bounded sets into bounded sets.

Remark 2.1. Assume that $A: X \rightarrow X^{*}$ is single-valued.

(i) If $A$ is monotone, then it is T-quasimonotone. 
(ii) If $A$ is B-pseudomonotone, then it is T-quasimonotone, but the inverse is not true in general. Indeed, consider the operator $A: X \longrightarrow X^{*}$ defined by

$$
A(x)= \begin{cases}0, & \text { if } \quad\|x\|<1, \\ J(x), & \text { if } \quad\|x\|=1,\end{cases}
$$

where $J$ is the duality mapping. We show that $A$ is a monotone operator. If $x, y$ are such that $\|x\|<1$ and $\|y\|<1$, it is clear that $A$ is monotone. If $\|x\|=1$ and $\|y\|<$ 1, we have $\langle A(x)-A(y), x-y\rangle=\langle J(x), x-y\rangle=\|x\|^{2}-\langle J(x), y\rangle \geq\|x\|^{2}-\|x\|\|y\|=$ $1-\|y\|>0$. If $\|x\|=\|y\|=1$, then $\langle A(x)-A(y), x-y\rangle=\langle J(x)-J(y), x-y\rangle \geq 0$. Thus, $A$ is monotone and consequently it is T-quasimonotone. Now, let us show that $A$ is not B-pseudomonotone. Let $u \in X$ such that $\|u\|=1$ be fixed and consider the sequence $u_{n}=\frac{n-1}{n} u, n \geq 1$. Then $u_{n} \rightarrow u$, therefore $u_{n} \rightarrow u$. On the other hand, we have $\left\langle A\left(u_{n}\right), u_{n}-u\right\rangle=0$. Thus, $\lim \sup \left\langle A\left(u_{n}\right), u_{n}-u\right\rangle=0 \leq 0$. Now, let $v=0$. Then $\liminf \left\langle A\left(u_{n}\right), u_{n}-v\right\rangle=0$. Therefore

$$
0=\liminf \left\langle A\left(u_{n}\right), u_{n}-v\right\rangle<1=\langle A(u), u-v\rangle .
$$

Thus $A$ is not B-pseudomonotone.

Now, let us recall some concepts for bifunctions; see, e.g., [14, 20, 22].

Definition 2.4. Let $K$ be a nonempty closed and convex subset of $X$. A real-valued bifunction $\Theta: K \times K \rightarrow \mathbb{R}$ is said to be

(i) monotone if, for all $x, y \in K, \Theta(x, y)+\Theta(y, x) \leq 0$;

(ii) pseudomonotone in the sense of Brézis (for short, B-pseudomonotne) if, for any sequence $\left(u_{n}\right)_{n \in \mathbb{N}} \subset K$ such that $u_{n} \rightarrow u$ in $X$ and liminf $\Theta\left(u_{n}, u\right) \geq 0$, we have limsup $\Theta\left(u_{n}, v\right) \leq \Theta(u, v)$ for all $v \in K$

(iii) quasimonotone in the topological sense (for short, T-quasimonotne) if, for any sequence $\left\{u_{n}\right\}_{n \in \mathbb{N}} \subset K$ such that $u_{n} \rightarrow u$ in $K$, we have

$$
\liminf _{n \rightarrow \infty} \Theta\left(u_{n}, u\right) \leq 0 .
$$

(iv) hemicontinuous (resp. upper hemicontinuous) if, for all $u, v, w \in K$ the functional $t \mapsto$ $\Theta(t u+(1-t) v, w)$ is continuous (resp. upper semicontinuous) on $[0,1]$.

Remark 2.2. (a) If the bifunction $\Theta: X \times X \longrightarrow \mathbb{R}$ is weakly upper semicontinuous with respect to the first argument, then it is B-pseudomonotne.

(b) If $A: X \longrightarrow X^{*}$ is B-pseudomonotne, then the bifunction $\Theta: X \times X \longrightarrow \mathbb{R}$ defined by $\Theta(u, v)=\langle A(u), v-u\rangle_{X}$ is B-pseudomonotne.

(c) If $\Theta_{1}, \Theta_{2}: X \times X \rightarrow \mathbb{R}$ are B-pseudomonotne such that $\Theta_{1}(u, u) \leq 0$ and $\Theta_{2}(u, u) \leq 0$ for all $u \in X$, then, $\Theta_{1}+\Theta_{2}$ is B-pseudomonotne; see [17].

In the following definition, we recall the notion of maximal monotonicity of bifunctions.

Definition 2.5. [14] Let $K$ be a nonempty closed convex subset of $X$, and let $\Phi: K \times K \longrightarrow \mathbb{R}$ be a real-valued bifunction such that $\Phi(u, u)=0$, for all $u \in K$. $\Phi$ is said to be maximal monotone if and only if, for every $u \in K$ and for every convex function $\varphi: K \longrightarrow \mathbb{R}$ with $\varphi(u)=0$,

$$
\Phi(v, u) \leq \varphi(v) \text { for all } v \in K \Longrightarrow 0 \leq \Phi(u, v)+\varphi(v) \text { for all } v \in K .
$$


Remark 2.3. The notion of the maximal monotonicity of bifunctions was introduced by Blum and Oettli [14] as an attempt to extend to bifunctions the notion of the maximal monotonicity of operators. Another notion of the maximal monotonicity of bifunctions was considered by Hadjisavvas and Khatibzadeh [21]. For a comparison between these two notions and some related properties, we refer to [21] and the references therein. For the examples and characterizations of maximal monotone bifunctions, we refer to [20, 26].

The following results from equilibrium problems theory will be crucial in the proof of our main theorems.

Theorem 2.1. [20, Theorem 2.3] Let $K$ be a nonempty, closed, and convex subset of a Banach space $X$, and let $\Phi, \Psi: K \times K \rightarrow \mathbb{R}$ be two real-valued bifunctions such that $\Phi(u, u)=\Psi(u, u)=$ 0 for all $u \in K$. Let $J: X \longrightarrow X^{*}$ be the duality mapping. Assume that

(i) $\Phi$ is monotone and maximal monotone;

(ii) $\Phi$ is weakly lower semicontinuous with respect to the second argument;

(iii) $\Phi$ and $\Psi$ are convex with respect to the second argument;

(iv) $\Psi$ is B-pseudomonotone;

(v) for each finite subset $A$ of $K$ and each $v$ in $K$ fixed, the function $u \longmapsto \Psi(u, v)$ is upper semicontinuous on $\operatorname{conv}(A)$;

(vi) (Coercivity) there exists a nonempty weakly compact subset $W$, such that for each $\varepsilon>0$ (small enough), there exists $B_{\varepsilon}$ a weakly compact and convex subset of $K$ satisfying the following: $\forall u \in K \backslash W$, there exists $v \in B_{\varepsilon}$ such that $\Psi(u, v)+\varepsilon\langle J(u), v-u\rangle<\Phi(v, u)$.

Then, there exists $u \in K$ such that $\Phi(u, v)+\Psi(u, v) \geq 0$, for all $v \in K$.

Theorem 2.2. [20] Let $K$ be a nonempty, closed, and convex subset of a Banach space $X$, and let $F, \Psi, \Xi: K \times K \rightarrow \mathbb{R}$ be real-valued bifunctions such that $F(u, u)=\Psi(u, u)=\Xi(u, u)=0$ for all $u \in K$. Let $J: X \longrightarrow X^{*}$ be the duality mapping. Assume that

(i) $\Phi$ is monotone and maximal monotone;

(ii) $\Phi$ is weakly lower semicontinuous with respect to the second argument;

(iii) $\Phi, \Psi$ and $\Xi$ and are convex with respect to the second argument;

(iv) $\Psi$ is T-quasiomonotone;

(v) for each $v$ in $K$ fixed, the function $u \longmapsto \Psi(u, v)$ is upper semicontinuous;

(vi) $\Xi$ is B-pseudomonotone;

(vii) for each finite subset $N$ of $K$ and each $v$ in $K$ fixed, the function $u \longmapsto \Xi(u, v)$ is upper semicontinuous on $\operatorname{conv}(N)$;

(viii) (coercivity) there exists a nonempty weakly compact subset $W$, such that, for each $\varepsilon>0$ (small enough), there exists $B_{\varepsilon}$ a weakly compact and convex subset of $K$ satisfying the following: $\forall u \in K \backslash W$, there exists $v \in B_{\varepsilon}$ such that $\Psi(u, v)+\Xi(u, v)+\varepsilon\langle J(u), v-u\rangle<$ $\Phi(v, u)$.

Then, there exists $u \in K$ such that $\Phi(u, v)+\Psi(u, v)+\Xi(u, v) \geq 0$, for all $v \in K$.

Remark 2.4. If $K$ is compact, then the coercivity condition (vi) in Theorem 2.1 and the coercivity condition (viii) in Theorem 2.2 can be dropped. When $X$ is a reflexive Banach space endowed with the weak topology $\sigma\left(X, X^{*}\right)$, theses coercivity conditions are satisfied if we suppose that there exists $v_{0} \in K$ such that $\Psi_{\varepsilon}\left(u, v_{0}\right) /\left\|u-v_{0}\right\| \rightarrow-\infty$, when $\left\|u-v_{0}\right\| \rightarrow+\infty$ uniformly in $\varepsilon>0$, where the bifunction $\Psi_{\varepsilon}$ takes respectively the following forms: $\Psi_{\varepsilon}(u, v):=$ 
$\Psi(u, v)+\varepsilon\langle J(u), v-u\rangle$ for Theorem 2.1, and $\Psi_{\varepsilon}(u, v):=\Psi(u, v)+\Xi(u, v)+\varepsilon\langle J(u), v-u\rangle$ for Theorem 2.2; see [20] for the details of the proof.

\section{Problem Statement and Main Results}

In this section, we provide the constrained problem under consideration in which the set of admissible elements is nonconvex. Our objective is to prove the existence of solutions.

Let $V$ be a reflexive Banach space, which is continuously and compactly embedded in a Hilbert space $H$. We denote by $i: V \rightarrow H$ the embedding operator, which is linear and compact. Let $\|\cdot\|_{V}$ and $\|\cdot\|_{H}$ be the norms in $V$ and $H$, respectively, the duality pairing between $V^{*}$ and $V$ is denoted by $\langle\cdot, \cdot\rangle$, and $\langle\cdot, \cdot\rangle_{H}$ stands for the inner product in $H$. Let $C_{0}$ be a nonempty set, which is closed and star-shaped with respect to a closed ball $\bar{B}\left(u_{0}, \rho\right)$ in $H$, where $u_{0} \in V$ and $\rho>0$. Let us denote by $C$ and $T_{C}(u)$ for $u \in C$ the realization of $C_{0}$ and $T_{C_{0}}(u)$ in $V$, i.e.,

$$
C:=C_{0} \cap V, \quad T_{C}(u):=T_{C_{0}}(u) \cap V,
$$

where $T_{C_{0}}(u)$ stands for the Clarke tangent cone of $C_{0}$ at $u$.

Let $A: V \rightarrow V^{*}$ be an operator, $\varphi: V \rightarrow \mathbb{R}$ be a functional and $j: H \rightarrow \mathbb{R}$ be a locally Lipschitz function. We consider the following problem:

$$
\left\{\begin{array}{l}
\text { Find } u \in C \text { such that } \\
\langle A(u), v-u\rangle+j^{0}(i(u) ; i(v)-i(u))+\varphi(v)-\varphi(u) \geq\langle f, v-u\rangle \text { for all } v \in u+T_{C}(u) .
\end{array}\right.
$$

On the data of problem (3.1), we assume the following hypotheses.

$\left[\mathrm{H}_{A}\right]$ The operator $A: V \rightarrow V^{*}$ is such that

(i) for each $N \in \mathscr{F}(V)$ and $v \in V$, the function $u \mapsto\langle A(u), v-u\rangle$ is upper semicontinuous on $\operatorname{conv}(N)$.

(ii) there exist $\alpha, \beta>0$ such that $\|A(u)\|_{V^{*}} \leq \alpha+\beta\|u\|_{V}$ for all $u \in V$;

(iii) there exist $a_{A}>0, b, c \in \mathbb{R}$ such that

$$
\langle A(u), u\rangle \geq a_{A}\|u\|_{V}^{2}+b\|u\|_{V}+c \text { for all } u \in V .
$$

$\left[\mathrm{H}_{j}\right] j: H \rightarrow \mathbb{R}$ is a locally Lipschitz function such that

$$
\|\partial j(u)\| \leq a_{0}+a_{j}\|u\|_{H} \text { for all } u \in H \text { with } a_{0}, a_{j} \geq 0 .
$$

$\left[\mathrm{H}_{\varphi}\right] \varphi: V \rightarrow \mathbb{R}$ is a convex and lower semicontinuous function.

$\left[\mathrm{H}_{C}\right] C \subset V$ is a closed set, which is star-shaped with respect to a ball $\bar{B}\left(u_{0}, \rho\right)$ in $H$, where $u_{0} \in V$ and $\rho>0$.

$\left[\mathrm{H}_{f}\right] f \in V^{*}$.

To study the existence of solutions of problem (3.1), we use a penalty method. Let $d: H \rightarrow \mathbb{R}$ be the distance function of the set $C_{0}$ defined by $d(u):=d_{C_{0}}(u)=\inf _{v \in C_{0}}\|v-u\|_{H}$ for $u \in H$. Let $\lambda>0$ represent a penalty parameter and consider the penalized problem corresponding to problem (3.1): Find $u_{\lambda} \in V$ such that

$$
\begin{aligned}
\left\langle A\left(u_{\lambda}\right)-f, v-u_{\lambda}\right\rangle & +j^{0}\left(i\left(u_{\lambda}\right) ; i(v)-i\left(u_{\lambda}\right)\right)+\varphi(v)-\varphi\left(u_{\lambda}\right) \\
& +\frac{1}{\lambda} d^{0}\left(u_{\lambda} ; v-u_{\lambda}\right) \geq 0, \quad \text { for all } v \in V .
\end{aligned}
$$

We have the following existence result for problem (3.1). 
Theorem 3.1. Assume that $\left[\mathrm{H}_{A}\right],\left[\mathrm{H}_{j}\right],\left[\mathrm{H}_{\varphi}\right],\left[\mathrm{H}_{C}\right]$, and $\left[\mathrm{H}_{f}\right]$ are satisfied, and $A: V \rightarrow V^{*}$ is B-pseudomonotone. Furthermore, suppose that the following smallness condition holds

$$
a_{A}-a_{j}\|i\|^{2}>0 .
$$

Then problem (3.1) has at least one solution.

Proof. The proof is divided into the following steps.

Step 1. We verify that, for each $\lambda>0$, penalized problem (3.2) has at least one solution $u_{\lambda} \in$ $V$. To this aim, we shall apply Theorem 2.1 by considering $K=V$, and the bifunctions $\Phi, \Psi: V \times V \rightarrow \mathbb{R}$ defined by $\Phi(u, v)=\varphi(v)-\varphi(u)+\langle f, u-v\rangle$ and $\Psi(u, v)=$ $\Psi_{1}(u, v)+\Psi_{2}(u, v)+\Psi_{3}(u, v)$, for $u, v \in V$, where $\Psi_{1}(u, v)=\langle A(u), v-u\rangle, \Psi_{2}(u, v)=$ $j^{0}(i(u) ; i(v)-i(u))$, and $\Psi_{3}(u, v)=\frac{1}{\lambda} d^{0}(u ; v-u)$. One can easily verify that $\Phi$ is monotone. From $\left[\mathrm{H}_{\varphi}\right]$ and $[20$, Lemma 2.4], we obtain that $\Phi$ is maximal monotone. Hence, the condition (i) of Theorem 2.1 is satisfied. By using condition $\left[\mathrm{H}_{\varphi}\right]$, we deduce that $\Phi$ is weakly lower semicontinuous with respect to the second argument, and hence the condition (ii) of Theorem 2.1 is satisfied.

On the other hand, by using $\left[\mathrm{H}_{\varphi}\right]$, we can easily verify that $\Phi$ is convex with respect to the second argument, and from Proposition 2.1 (i), we deduce that $\Psi$ is convex with respect to the second argument. Hence, the condition (iii) of Theorem 2.1 is satisfied.

Now, let us verify that $\Psi$ is B-pseudomonotone. As $A$ is B-pseudomonotone, then $\Psi_{1}$ is B-pseudomonotone. On the other hand, since $i: V \rightarrow H$ is linear and compact, we deduce, thanks to Proposition 2.1 (ii), that $\Psi_{2}(\cdot, v)$ is weakly upper semicontinuous for each $v \in V$. Hence, from Remark 2.2 (a), we obtain that $\Psi_{2}$ is B-pseudomonotone. Similarly, one can verify that $\Psi_{3}$ is B-pseudomonotone. Therefore, by Remark 2.2 (c), we deduce that the bifunction $\Psi$ is B-pseudomonotone as being the sum of bifunctions, which are B-pseudomonotone. Thus, the condition (iv) of Theorem 2.1 is satisfied. For the condition (v) of Theorem 2.1, we note that $\left[\mathrm{H}_{A}\right]$ (i) implies that $\Psi_{1}(\cdot, v)$ is upper semicontinuous on $\operatorname{conv}(N)$ for each $N \in \mathscr{F}(V)$. Moreover, as $\Psi_{2}(\cdot, v)$ and $\Psi_{3}(\cdot, v)$ are weakly upper semicontinuous on $V$, it follows that they are upper semicontinuous on $\operatorname{conv}(N)$ for each $N \in \mathscr{F}(V)$. Therefore, $\Psi(\cdot, v)$ is upper semicontinuous on $\operatorname{conv}(N)$ for each $N \in \mathscr{F}(V)$ and hence the condition (v) of Theorem 2.1 is satisfied.

Now, let us verify that the coercivity condition (vi) of Theorem 2.1 holds. To this aim, from Remark 2.4, we need to verify that, for some $v_{0} \in V$,

$$
\Psi_{\varepsilon}\left(u, v_{0}\right) /\left\|u-v_{0}\right\| \rightarrow-\infty \text { uniformly in } \varepsilon>0, \text { when }\left\|u-v_{0}\right\| \rightarrow+\infty,
$$

where $\Psi_{\varepsilon}(u, v):=\Psi(u, v)+\varepsilon\langle J(u), v-u\rangle$. We take $v_{0}=\mathbf{0}$. Then

$$
\begin{aligned}
\Psi_{\varepsilon}(u, \mathbf{0}) & =-\langle A(u), u\rangle+j^{0}(i(u) ;-i(u))+\frac{1}{\lambda} d^{0}(u ;-u)-\varepsilon\|u\|^{2} \\
& \leq-\langle A(u), u\rangle+j^{0}(i(u) ;-i(u))+\frac{1}{\lambda} d^{0}(u ;-u) .
\end{aligned}
$$

From Proposition 2.2 (ii), we have that

$$
j^{0}(i(u) ;-i(u))=\max \left\{\left\langle u^{*}, i(u)\right\rangle: u^{*} \in \partial j(i(u))\right\} .
$$


Thus, by using $\left[\mathrm{H}_{j}\right]$, we obtain

$$
\begin{aligned}
j^{0}(i(u) ;-i(u)) & \leq\left(a_{0}+a_{j}\|i(u)\|_{H}\right)\|i(u)\|_{H} \\
& \leq a_{0}\|i\|\|u\|_{V}+a_{j}\|i\|^{2}\|u\|_{V}^{2} .
\end{aligned}
$$

On the other hand, as the function $d$ is Lipschitz continuous with Lipschitz constant $k=1$, we deduce from Proposition 2.2 (i) that

$$
d^{0}(u ;-u) \leq\|u\|_{H} \leq\|i\|\|u\|_{V}, \text { for all } v \in V .
$$

Hence, by (3.6), (3.7) and $\left[\mathrm{H}_{A}\right](\mathrm{iii})$, we deduce from (3.5) that

$$
\Psi_{\varepsilon}(u, \mathbf{0}) \leq-\left(a_{A}-a_{j}\|i\|^{2}\right)\|u\|_{V}^{2}+\left(\frac{1}{\lambda}\|i\|-b+a_{0}\|i\|\right)\|u\|_{V}-c .
$$

By the smallness condition (3.3), we derive from the previous inequality that

$$
\Psi_{\varepsilon}(u, \mathbf{0}) /\|u\|_{V} \rightarrow-\infty
$$

uniformly in $\varepsilon>0$ when $\|u\| \rightarrow+\infty$. Thus, (3.5) holds and hence the coercivity condition (vi) of Theorem 2.1 is satisfied. Therefore, from Theorem 2.1, we deduce that, for each $\lambda>0$, penalized problem (3.2) has a solution $u_{\lambda} \in V$.

Step 2. We verify that there exists $M>0$ such that $\left\|u_{\lambda}\right\|_{V} \leq M$ for all $\lambda>0$. Let us take $v=u_{0}$ in relation (3.2), where $u_{0} \in V$ is the center of the ball $\bar{B}\left(u_{0}, \rho\right)$. Then,

$$
\begin{array}{r}
\left\langle A\left(u_{\lambda}\right)-f, u_{0}-u_{\lambda}\right\rangle+j^{0}\left(i\left(u_{\lambda}\right) ; i\left(u_{0}\right)-i\left(u_{\lambda}\right)\right)+\varphi\left(u_{0}\right)-\varphi\left(u_{\lambda}\right) \\
+\frac{1}{\lambda} d^{0}\left(u_{\lambda} ; u_{0}-u_{\lambda}\right) \geq 0 .
\end{array}
$$

It follows that

$$
\begin{array}{r}
\left\langle A\left(u_{\lambda}\right), u_{0}\right\rangle+\left\langle f, u_{\lambda}-u_{0}\right\rangle+j^{0}\left(i\left(u_{\lambda}\right) ; i\left(u_{0}\right)-i\left(u_{\lambda}\right)\right)+\varphi\left(u_{0}\right)-\varphi\left(u_{\lambda}\right) \\
+\frac{1}{\lambda} d^{0}\left(u_{\lambda} ; u_{0}-u_{\lambda}\right) \geq\left\langle A\left(u_{\lambda}\right), u_{\lambda}\right\rangle
\end{array}
$$

As $\varphi$ is convex and lower semicontinuous, it is bounded from below by an affine function; see, e.g., [27, Proposition 5.2.25]. Hence, there exist $z^{*} \in V^{*}$ and $\tau \in \mathbb{R}$ such that

$$
\varphi(v) \geq\left\langle z^{*}, v\right\rangle+\tau, \text { for all } v \in V
$$

This implies that

$$
-\varphi\left(u_{\lambda}\right) \leq-\left\langle z^{*}, u_{\lambda}\right\rangle-\tau \leq\left\|z^{*}\right\|_{V^{*}}\left\|u_{\lambda}\right\|_{V}+|\tau| .
$$

On the other hand, from Proposition 2.2 (ii) and $\left[\mathrm{H}_{j}\right]$, we obtain that

$$
\begin{aligned}
j^{0}\left(i\left(u_{\lambda}\right) ; i\left(u_{0}\right)-i\left(u_{\lambda}\right)\right) & \leq\left(a_{0}+a_{j}\left\|i\left(u_{0}\right)-i\left(u_{\lambda}\right)\right\|_{H}\right)\left\|i\left(u_{0}\right)-i\left(u_{\lambda}\right)\right\|_{H} \\
& \leq a_{0}\|i\|\left\|u_{0}-u_{\lambda}\right\|_{V}+a_{j}\|i\|^{2}\left\|u_{0}-u_{\lambda}\right\|_{V}^{2} .
\end{aligned}
$$

Hence, using (3.8), (3.9)-(3.11), and $\left[\mathrm{H}_{A}\right]$ (ii)-(iii), we obtain the following estimation

$$
m_{0}+m_{1}\left\|u_{\lambda}\right\|_{V}+\frac{1}{\lambda} d^{0}\left(u_{\lambda} ; u_{0}-u_{\lambda}\right) \geq\left(a_{A}-a_{j}\|i\|^{2}\right)\left\|u_{\lambda}\right\|_{V}^{2},
$$

where

$$
\begin{aligned}
& m_{0}=\left[\alpha+\|f\|_{V^{*}}+a_{0}\|i\|+a_{j}\|i\|\left\|u_{0}\right\|_{V}\right]\left\|u_{0}\right\|_{V}+\left|\varphi\left(u_{0}\right)\right|+|\tau|+|c| \\
& m_{1}=\left[\beta+2 a_{j}\|i\|^{2}\right]\left\|u_{0}\right\|_{V}+\|f\|_{V^{*}}+a_{0}\|i\|+\left\|z^{*}\right\|_{V^{*}}+|b| .
\end{aligned}
$$


On the other hand, by Lemma 2.1, we obtain that $d^{0}\left(u_{\lambda} ; u_{0}-u_{\lambda}\right) \leq 0$. Hence, from (3.12), we deduce that

$$
m_{0}+m_{1}\left\|u_{\lambda}\right\|_{V} \geq\left(a_{A}-a_{j}\|i\|^{2}\right)\left\|u_{\lambda}\right\|_{V}^{2}
$$

which implies, thanks to the smallness condition (3.3), that $\left\{u_{\lambda}\right\}_{\lambda>0}$ is bounded. Therefore, there exists $M>0$ such that $\left\|u_{\lambda}\right\|_{V} \leq M$, for all $\lambda>0$.

Step 3. Let us verify that the solution $u_{\lambda} \in V$ of penalized problem (3.2) belongs to $C$ for $\lambda>0$ sufficiently small. Form Lemma 2.1 , we have that $d_{C}^{0}\left(u_{\lambda} ; u_{0}-u_{\lambda}\right) \leq-d_{C}\left(u_{\lambda}\right)-\rho$ for $u_{\lambda} \notin C$. Hence, by (3.12), we deduce that, for $u_{\lambda} \notin C$,

$$
m_{0}+m_{1} M-\frac{1}{\lambda} \rho \geq \frac{1}{\lambda} d_{C}\left(u_{\lambda}\right) \geq 0,
$$

which implies that $\lambda \geq \frac{\rho}{m_{0}+m_{1} M}$. Let us set $\lambda_{0}:=\frac{\rho}{m_{0}+m_{1} M}$. From what precedes, we conclude that $u_{\lambda} \in C$ for all $\lambda \in\left(0, \lambda_{0}\right)$.

Step 4. Let us verify that, for $\lambda \in\left(0, \lambda_{0}\right), u_{\lambda}$ is a solution of problem (3.1). From Step 3, we have that $u_{\lambda} \in C$ for any $\lambda \in\left(0, \lambda_{0}\right)$. As $u_{\lambda}$ is a solution of penalized problem (3.2), for all $v \in V$, we have

$$
\begin{array}{r}
\left\langle A\left(u_{\lambda}\right)-f, v-u_{\lambda}\right\rangle+j^{0}\left(i\left(u_{\lambda}\right) ; i(v)-i\left(u_{\lambda}\right)\right)+\varphi(v)-\varphi\left(u_{\lambda}\right) \\
+\frac{1}{\lambda} d^{0}\left(u_{\lambda} ; v-u_{\lambda}\right) \geq 0 .
\end{array}
$$

Let us take $v \in u_{\lambda}+T_{C}\left(u_{\lambda}\right)$. It follows that $v-u_{\lambda} \in T_{C}\left(u_{\lambda}\right)$ and hence $d^{0}\left(u_{\lambda} ; v-u_{\lambda}\right)=$ 0 . Therefore, we obtain from (3.15) that

$$
\begin{array}{r}
\left\langle A\left(u_{\lambda}\right)-f, v-u_{\lambda}\right\rangle+j^{0}\left(i\left(u_{\lambda}\right) ; i(v)-i\left(u_{\lambda}\right)\right)+\varphi(v)-\varphi\left(u_{\lambda}\right) \geq 0, \\
\forall v \in u_{\lambda}+T_{C}\left(u_{\lambda}\right) .
\end{array}
$$

Thus, for $\lambda \in\left(0, \lambda_{0}\right), u_{\lambda}$ is a solution of problem (3.1)

Theorem 3.2. Assume that $\left[\mathrm{H}_{j}\right],\left[\mathrm{H}_{\varphi}\right],\left[\mathrm{H}_{C}\right],\left[\mathrm{H}_{f}\right]$ hold and $A: V \rightarrow V^{*}$ is $\mathrm{T}$-quasimonotone and satisfies $\left[\mathrm{H}_{A}\right]$ (ii)-(iii). Furthermore, suppose that 3.3 holds. Then problem (3.1) has at least one solution.

Proof. First, we verify that, for each $\lambda>0$, penalized problem (3.2) has at least one solution $u_{\lambda} \in V$. To this aim, we shall apply Theorem 2.2 by considering $K=V$, and the bifunctions $\Phi, \Psi, \Xi: V \times V \rightarrow \mathbb{R}$ defined for $u, v \in V$ by $\Phi(u, v)=\varphi(v)-\varphi(u)+\langle f, u-v\rangle$, $\Psi(u, v)=\langle A(u), v-u\rangle$, and $\Xi(u, v)=\Psi_{2}(u, v)+\Psi_{3}(u, v)$, where $\Psi_{2}(u, v)=j^{0}(i(u) ; i(v)-i(u))$ and $\Psi_{3}(u, v)=\frac{1}{\lambda} d^{0}(u ; v-u)$. We proceed similarly as in Step 1 of the proof of Theorem 3.1 to show that all the conditions of Theorem 2.2 are satisfied. The rest of the proof is similar to the one of Theorem 3.1 by proceeding similarly as in Step 2, Step 3, and Step 4.

Remark 3.1. In comparison with recent studies in literature on the problem considered in this paper, we can see that by our approach we considerably improve Theorems 1 and 2 in [13]. 


\section{Nonconvex SEmipermeability Problem}

In this section, we give an illustration of the approach developed in this paper to study the boundary semipermeability problems whose weak formulation leads to a (nonconvex) constrained variational-hemivariational inequality. Such problems arise in several situations in hydraulic, flow problems through porous media and electrostatics; see, e.g., [2, 3].

Semipermeability problems were first studied by Duvaut and Lions [28] in the context of convexity, i.e., maximal monotone boundary relations. These relations lead to variational inequalities associated to the Laplacian operator and arise in heat conduction, electrostatics, and flow problems through porous media. Semipermeability problems with nonmonotone boundary conditions are met in certain categories of temperature control problems in heat conduction, or potential control and pressure control in electrostatics and hydraulics, respectively. In such problems, the nonmonotone and multivalued semipermeability conditions are expressed by the generalized gradient of Clarke for nonconvex superpotential. These models can not be handled by mean of variational inequalities and they need a hemivariational inequality approach; see, e.g., $[11,29,30]$.

We consider a semipermeability model for the stationary heat conduction problem which a particular form has been recently studied by Migórski and Fengzhen [13]. Let $\Omega$ be an open domain in $\mathbb{R}^{d}$ with a Lipschitz continuous boundary $\Gamma:=\partial \Omega$. The boundary $\Gamma$ is composed of three sets $\bar{\Gamma}_{1}, \bar{\Gamma}_{2}$ and $\bar{\Gamma}_{3}$, with disjoint relatively open sets $\Gamma_{1}, \Gamma_{2}$, and $\Gamma_{3}$ such that meas $\left(\Gamma_{1}\right)>0$. We are interested in a mathematical model with a nonconvex constraint set, that describes the temperature of a body occupying volume $\Omega$. The problem under consideration reads as follows:

Problem $(\mathscr{P}):$ Find a temperature $u: \Omega \rightarrow \mathbb{R}$ such that

$$
\begin{array}{ccc}
A(u)+\partial j_{1}(u)+\partial g_{1}(u) \ni f_{1}+g & \text { in } & \Omega, \\
u=0 & \text { on } & \Gamma_{1}, \\
\frac{\partial u}{\partial v_{A}}+\partial j_{2}(u) \ni f_{2} & \text { on } & \Gamma_{2}, \\
\frac{\partial u}{\partial v_{A}}+\partial g_{2}(u) \ni f_{3} & \text { on } & \Gamma_{3},
\end{array}
$$

where $A: V \rightarrow V^{*}$ is a given mapping, $V=\left\{v \in H^{1}(\Omega): v=0\right.$ on $\left.\Gamma_{1}\right\}, \frac{\partial u}{\partial v_{A}}$ denotes the outward conormal derivative with respect to $A$ on $\Gamma$ and represents the heat flux through a part of the boundary, $f_{1}$ is a given heat source per unit volume in $\Omega, f_{2}$ and $f_{3}$ are the heat flux through $\Gamma_{2}$ and $\Gamma_{3}$, respectively. Here, the mappings $j_{1}$ and $g_{1}$ describe the interior semipermeability phenomena, while $j_{2}$ and $g_{2}$ provide the boundary semipermeability relations in the subdifferential form. Further, $g \in V$ is a function of the temperature $u$, introduced in order to incorporate constraints to the model. We will assume that $u \in C$ and $-g \in N_{C}(u)$, where $C$ denotes a nonempty closed subset of $V$ and $N_{C}(u)$ is the Clarke's normal cone to $C$ at $u$. Consequently,

$$
\langle g, w\rangle \geq 0 \quad \text { for all } w \in T_{C}(u),
$$

where $T_{C}(u)$ is the Clarke's tangent cone of $C$ at $u$. The set $C$, which can be convex or nonconvex, can be used to introduce additional constraints for the temperature (or potential control and pressure control in electrostatic and hydraulic models).

We denote by $i: V \rightarrow L^{2}(\Omega)$ the embedding operator and by $\gamma: V \rightarrow L^{2}(\Gamma)$ the trace operator. It is well known that both operators are linear and compact. 
The assumptions on the data of the problem $(\mathscr{P})$ are as the following:

$\left[\mathrm{H}_{a}\right] A: V \rightarrow V^{*}$ is an operator such that $A(v)(x)=-\sum_{i=1}^{d} \frac{\partial}{\partial x_{i}} a_{i}(x, v(x), \nabla v(x))$ where the coefficients $a_{i}: \Omega \times \mathbb{R} \times \mathbb{R}^{d} \rightarrow \mathbb{R}, i=1, \ldots, d$ satisfy the following hypotheses:

(i) Carathéodory and Growth Condition: Each $a_{i}(x, s, \xi)$ satisfies Carathéodory conditions, i.e., is measurable in $x \in \Omega$ for all $(s, \xi) \in \mathbb{R} \times \mathbb{R}^{d}$ and continuous in $(s, \xi)$ for a.e. $x \in \Omega$. A constant $m_{0}>0$ and a function $k_{0} \in L^{2}(\Omega)$ exist so that

$$
\left|a_{i}(x, s, \xi)\right| \leq m_{0}\left[k_{0}(x)+|s|+|\xi|\right]
$$

for a.e. $x \in \Omega$ and for all $(s, \xi) \in R \times \mathbb{R}^{d}$, with $|\xi|$ denoting the Euclidian norm of the vector $\xi$.

(ii) Monotonicity Type Condition: The coefficients $a_{i}$ satisfy a monotonicity condition with respect to $\xi$ in the form

$$
\sum_{i=1}^{d}\left(a_{i}(x, s, \xi)-a_{i}(x, s, \eta)\right)\left(\xi_{i}-\eta_{i}\right) \geq 0
$$

for a.e. $x \in \Omega$, for all $s \in \mathbb{R}$, and for all $\xi, \eta \in \mathbb{R}^{d}$.

(iii) Coercivity Type Condition:

$$
\sum_{i=1}^{d} a_{i}(x, s, \xi) \xi_{i} \geq m_{1}\left[|s|^{2}+|\xi|^{2}\right],
$$

for all $(s, \xi) \in \mathbb{R} \times \mathbb{R}^{d}$, a.e. $x \in \Omega$ with some constant $m_{1}>0$.

$\left[\mathrm{H}_{j_{1}}\right] j_{1}: \mathbb{R} \rightarrow \mathbb{R}$ is a locally Lipschitz function such that

$$
\left\|\partial j_{1}(r)\right\| \leq b_{0}+b_{1}|r| \text { for all } r \in \mathbb{R} \text { with } b_{0}, b_{1} \geq 0 \text {. }
$$

$\left[\mathrm{H}_{j_{2}}\right] j_{2}: \mathbb{R} \rightarrow \mathbb{R}$ is a locally Lipschitz function such that

$$
\left\|\partial j_{2}(r)\right\| \leq c_{0}+c_{1}|r| \text { for all } r \in \mathbb{R} \text { with } c_{0}, c_{1} \geq 0 .
$$

$\left[\mathrm{H}_{g_{1}}\right] g_{1}: \mathbb{R} \rightarrow \mathbb{R}$ is a convex and lower semicontinuous function such that

$$
\left\|\partial g_{1}(r)\right\| \leq \alpha_{0}+\alpha_{1}|r| \text { for all } r \in \mathbb{R} \text { with } \alpha_{0}, \alpha_{1} \geq 0 .
$$

$\left[\mathrm{H}_{g_{2}}\right] g_{2}: \mathbb{R} \rightarrow \mathbb{R}$ is a convex and lower semicontinuous function such that

$$
\left\|\partial g_{2}(r)\right\| \leq \beta_{0}+\beta_{1}|r| \text { for all } r \in \mathbb{R} \text { with } \beta_{0}, \beta_{1} \geq 0 \text {. }
$$

$\left[\mathrm{H}_{f}\right] f_{1} \in L^{2}(\Omega), f_{2} \in L^{2}\left(\Gamma_{2}\right), f_{3} \in L^{2}\left(\Gamma_{3}\right)$.

[H $\left.\mathrm{H}_{0}\right]$ Smallness Condition: $m_{1}>b_{1}\|i\|^{2}+c_{1}\|\gamma\|^{2}$.

$\left[\mathrm{H}_{C}\right] C \subset V$ is a closed set, which is star-shaped with respect to a ball $\bar{B}\left(u_{0}, \rho\right)$ with $u_{0} \in V$ and $\rho>0$.

The differential operator $A$ generates a mapping from $V$ to its dual defined by

$$
\langle A(v), w\rangle=\int_{\Omega} \sum_{i=1}^{d} a_{i}(x, v(x), \nabla v(x)) \frac{\partial w}{\partial x_{i}} d x
$$

for all $v, w \in V$. By using [31, Theorem 2.109], we obtain from $\left[\mathrm{H}_{a}\right](\mathrm{i})$-(ii) that $\left[\mathrm{H}_{A}\right]$ is satisfied.

Under the hypotheses above, by a standard procedure, we obtain the following weak formulation of the problem $(\mathscr{P})$. 
Problem $\left(\mathscr{P}_{w}\right):$ Find $u \in V$ such that

$$
\begin{array}{r}
\langle A(u)-f, v-u\rangle+\int_{\Omega} j_{1}^{0}(i(u) ; i(v)-i(u)) d x+\int_{\Gamma_{2}} j_{2}^{0}(\gamma(u) ; \gamma(v)-\gamma(u)) d \Gamma \\
+\int_{\Omega}\left[g_{1}(i(v))-g_{1}(i(u))\right] d x+\int_{\Gamma_{3}}\left[g_{2}(\gamma(v))-g_{2}(\gamma(u))\right] d \Gamma \geq\langle g, v-u\rangle, \quad \text { for all } v \in V,
\end{array}
$$

where $f \in V^{*}$ is defined by

$$
\langle f, v\rangle=\int_{\Omega} f_{1}(x) i(v(x)) d x+\int_{\Gamma_{2}} f_{2}(x) \gamma(v(x)) d x+\int_{\Gamma_{3}} f_{3}(x) \gamma(v(x)) d x, \text { for all } v \in V .
$$

Under the condition (4.1), problem $\left(\mathscr{P}_{w}\right)$ takes the form:

$$
\left\{\begin{array}{l}
\text { Find } u \in C \text { such that } \\
\langle A(u)-f, v-u\rangle+\int_{\Omega} j_{1}^{0}(i(u) ; i(v)-i(u)) d x+\int_{\Gamma_{2}} j_{2}^{0}(\gamma(u) ; \gamma(v)-\gamma(u)) d \Gamma \\
+\int_{\Omega}\left[g_{1}(i(v))-g_{1}(i(u))\right] d x+\int_{\Gamma_{3}}\left[g_{2}(\gamma(v))-g_{2}(\gamma(u))\right] d \Gamma \geq 0, \text { for all } v \in u+T_{C}(u) .
\end{array}\right.
$$

We consider the functionals $J_{1}, J_{2}, \varphi_{1}, \varphi_{2}: V \rightarrow \mathbb{R}$ defined for all $v \in V$ by

$$
\begin{array}{ll}
J_{1}(v)=\int_{\Omega} j_{1}(i(v)) d x, & J_{2}(v)=\int_{\Gamma_{2}} j_{2}(\gamma(v)) d \Gamma, \\
\varphi_{1}(v)=\int_{\Omega} g_{1}(i(v)) d x, & \varphi_{2}(v)=\int_{\Gamma_{2}} g_{2}(\gamma(v)) d \Gamma .
\end{array}
$$

Let us set $J=J_{1}+J_{2}$ and $\varphi=\varphi_{1}+\varphi_{2}$. From [1, Proposition 3.37 (i)] and [1, Proposition 3.47 (iv)], we obtain that

$$
\begin{aligned}
J^{0}(u ; v-u) & \leq J_{1}^{0}(u ; v-u)+J_{2}^{0}(u ; v-u) \\
& \leq \int_{\Omega} j_{1}^{0}(i(u) ; i(v)-i(u)) d x+\int_{\Gamma_{2}} j_{2}^{0}(\gamma(u) ; \gamma(v)-\gamma(u)) d \Gamma .
\end{aligned}
$$

Therefore, by using the previous inequality, we see that the existence of solutions of problem (4.3) is obtained by solving the following problem:

$$
\left\{\begin{array}{l}
\text { Find } u \in C \text { such that } \\
\langle A(u)-f, v-u\rangle+J^{0}(u ; v-u)+\varphi(v)-\varphi(u) \geq 0, \text { for all } v \in u+T_{C}(u) .
\end{array}\right.
$$

Applying Theorem 3.1 to problem (4.5), we obtain the following result.

Theorem 4.1. If the hypotheses $\left[\mathrm{H}_{a}\right],\left[\mathrm{H}_{j_{1}}\right],\left[\mathrm{H}_{j_{2}}\right],\left[\mathrm{H}_{g_{1}}\right],\left[\mathrm{H}_{g_{2}}\right],\left[\mathrm{H}_{f}\right],\left[\mathrm{H}_{0}\right]$ and $\left[\mathrm{H}_{C}\right]$ hold, then problem (4.5) admits at least one solution.

Remark 4.1. By the approach developed in this paper, we considerably improve the study of the Problem 3 in [13] since we do not need any conditions of the type

$$
(\partial j(r)-\partial j(s))(r-s) \geq-k|r-s|^{2} \text { for all } r, s \in \mathbb{R} \text { with } k \geq 0
$$

on $j_{1}$ and $j_{2}$.

\section{Acknowledgements}

The authors are grateful to the referees for their useful suggestions which improved the contents of this paper. The research of J.C. Yao was partially supported by the National Natural Science Foundation of China (1217011852). 


\section{REFERENCES}

[1] S. Migórski, A. Ochal, M. Sofonea, Nonlinear Inclusions and Hemivariational Inequalities. Models and Analysis of Contact Problems, Advances in Mechanics and Mathematics 26, Springer, New York, 2013.

[2] Z. Naniewicz, P. D.Panagiotopoulos, Mathematical Theory of Hemivariational Inequalities and Applications, Marcel Decker, New York, 1995.

[3] P.D. Panagiotopoulos, Hemivariational Inequalities, Applications in Mechanics and Engineering, Springer, Berlin, 1993.

[4] L.V. Nguyen, X. Qin, The minimal time function associated with a collection of sets, ESAIM Control Optim. Calc. Var. 26 (2020), 93.

[5] M. Sofonea, S. Migórski, Variational-Hemivariational Inequalities with Applications, Pure Appl. Math., Chapman \& Hall/CRC Press, Boca Raton-London, 2018.

[6] O. Chadli, J. Gwinner, N. Ovcharova, On semicoercive variational-hemivariational inequalities - existence, approximation, and regularization, Vietnam. J. Math. 46 (2018), 329-342.

[7] N. Ovcharova, L. Banz, Coupling regularization and adaptive hp-BEM for the solution of a delamination problem, Numer. Math. 137 (2017), 303-337.

[8] Z. Naniewicz, Hemivariational inequality approach to constrained problems for star-shaped admissible sets, J. Optim. Theory Appl. 83 (1994), 97-112.

[9] D. Goeleven, On the hemivariational inequality approach to nonconvex constrained problems in the theory of von Kárman plates, Z. Angew. Math. Mech. 75 (1995), 861-866.

[10] D. Goeleven, Noncoercive hemivariational inequality approach to constrained problems for star-shaped admissible sets, J. Global Optim. 9 (1996), 121-140.

[11] L. Gasínski, Z. Liu, S. Migórski, A. Ochal, Z. Peng, Hemivariational inequality approach to evolutionary constrained problems on star-shaped sets, J. Optim. Theory Appl. 164 (2015), 514-533.

[12] Z. Peng, L. Gasiński, S. Migórski, A. Ochal, A class of evolution variational inequalities with nonconvex constraints, Optimization, 68 (2019), 1881-1895.

[13] S. Migórski, L. Fengzhen, Constrained variational-hemivariational inequalities on nonconvex star-shaped sets, Mathematics, 8 (2020), 1824. DOI: 10.3390/math8101824.

[14] E. Blum, W. Oettli, From optimization and variational inequalities to equilibrium problems, Math. Stud. 63 (1994), 123-145.

[15] S.Y. Cho, A monotone Bregan projection algorithm for fixed point and equilibrium problems in a reflexive Banach space, Filomat, 34 (2020), 1487-1497.

[16] X. Qin, S.Y. Cho, Convergence analysis of a monotone projection algorithm in reflexive Banach spaces, Acta Math. Sci. 37 (2017), 488-502.

[17] L. Liu, On the resolution of variational inequality problems with a double-hierarchical structure, J. Nonlinear Convex Anal. 21 (2020), 377-386.

[18] S.Y. Cho, A convergence theorem for generalized mixed equilibrium problems and multivalued asymptotically nonexpansive mappings, J. Nonlinear Convex Anal. 21 (2020), 1017-1026.

[19] B. Tan, S.Y. Cho, Inertial projection and contraction methods for pseudomonotone variational inequalities with non-Lipschitz operators and applications, Appl. Anal. (2021), doi: 10.1080/00036811.2021.1979219.

[20] O. Chadli, Q.H. Ansari, J.-C. Yao, Mixed equilibrium problems and anti-periodic solutions for nonlinear evolution equations, J. Optim. Theory Appl. 168 (2016), 410-440.

[21] N. Hadjisavvas, H. Khatibzadeh, Maximal monotonicity of bifunctions, Optimization 59 (2010), 147-160.

[22] J. Gwinner, A note on pseudomonotone functions, regularization, and relaxed coerciveness, Nonlinear Anal. 30 (1997), 4217-4227.

[23] H. Brézis, Equations et inéquations nonlinéaires dans les espaces vectoriels en dualité. Ann. Inst. Fourier 18 (1968), 115-175.

[24] F.H. Clarke, Optimization and Nonsmooth Analysis, Wiley, New York, 1983.

[25] F.H. Clarke, Functional Analysis, Calculus of Variations and Optimal Control, Springer-Verlag, London, 2013.

[26] O. Chadli, Q.H. Ansari, S. Al-Homidan, Existence of solutions for nonlinear implicit differential equations: an equilibrium problem approach, Numer. Funct. Anal. Optim. 37 (2016), 1385-1419. 
[27] Z. Denkowski, S. Migórski, N.S. Papageorgiou, An Introduction to Nonlinear Analysis: Theory, Kluwer Academic Publishers, Boston, MA, USA, 2003.

[28] G. Duvaut, J.L. Lions, Inequalities in Mechanics and Physics, Springer, Berlin, 1976.

[29] P.D. Panagiotopoulos, Nonconvex problems of semipermeable media and related topics, Z. Angew. Math. Mech. 65 (1985), 29-36.

[30] S. Migórski, A. Ochal, Boundary hemivariational inequality of parabolic type, Nonlinear Anal. 57 (2004), 579-596.

[31] S. Carl, VK. Le, D. Motreanu, Nonsmooth Variational Problems and Their Inequalities: Comparison Principles and Applications, Springer, New York, 2007. 